\title{
Epidemiology of acute kidney injury in intensive care units in Beijing: the multi- center BAKIT study
}

\author{
Li Jiang ${ }^{1 \dagger}$, Yibing Zhu ${ }^{12^{*}+}$, Xuying Luo ${ }^{1,3 \dagger}$, Ying Wen ${ }^{1,4}$, Bin Du ${ }^{5}$, Meiping Wang ${ }^{1,6}$, Zhen Zhao ${ }^{1}$, Yanyan Yin ${ }^{1,7}$, \\ Bo Zhu', Xiuming $\mathrm{Xi}^{1{ }^{*}}$ and The Beijing Acute Kidney Injury Trial (BAKIT) workgroup
}

\begin{abstract}
Background: Acute kidney injury (AKI) commonly occurs in intensive care units (ICUs), leading to adverse clinical outcomes and increasing costs. However, there are limited epidemiological data of AKI in the critically ill in Beijing, China.

Methods: In this prospective cohort study in 30 ICUs, we screened the patients up to 10 days after ICU admission. Characteristics and outcomes were compared between AKI and non-AKI, renal replacement therapy (RRT) and non-RRT patients. Nomograms of logistic regression and Cox regression were performed to examine potential risk factors for AKI and mortality.

Results: A total of 3107 patients were included in the final analysis. The incidence of AKI was 51.0\%; stages 1 to 3 accounted for 23.1, 11.8, and 15.7\%, respectively. The majority (87.6\%) of patients with AKI developed AKI on the first 4 days after admission to the ICU. A total of 281 patients were treated with RRT. Continuous RRT with predilution, citrate for anticoagulation and femoral vein for vascular access was the most common RRT pattern (29.9\%, 84 of 281). Patients with AKI were associated with longer ICU-LOS and higher mortality and costs $(P<0.001)$. In patients treated with RRT, 78.6 and $28.5 \%$ of RRTs were dependent on the 7 th and 28th days, respectively. The 28 day mortalities of non-AKI, AKI stages 1-3, and septic shock patients were 6.83, 15.04, 27.99, 45.18 and 36.5\%, respectively.

Conclusions: Approximately half of our ICU patients experienced AKI. The majority of patients with AKI developed AKI during the first 4 days after admission to the ICU. Continuous RRT with predilution, citrate for anticoagulation and femoral vein for vascular access was the most common RRT pattern in our ICUs. AKI was associated with a higher mortality and costs, incomplete kidney recovery and s series of adverse outcomes.
\end{abstract}

Keywords: Acute kidney injury, Renal replacement therapy, Critical care medicine, Mortality, Epidemiology

\section{Background}

Acute kidney injury (AKI) is a life-threatening disease and global health burdens with increasing incidence in both developed and developing countries [1, 2]. AKI commonly occurs in the intensive care unit (ICU), and is caused by multiple risk factors, leading to adverse clinical outcomes, increasing costs, and the development of chronic kidney disease (CKD) [3-8]. The definition of AKI has evolved from the Risk, Injury, Failure, Loss, End-

\footnotetext{
*Correspondence: yiyi_bingbing@163.com; xxm2937@sina.com

†Li Jiang, Yibing Zhu and Xuying Luo contributed equally to this work. ${ }^{1}$ Department of Critical Care Medicine, Fuxing Hospital, Capital Medical University, 20A Fuxingmenwai Street, Xicheng District, Beijing 100038, China Full list of author information is available at the end of the article
}

stage (RIFLE) criteria and the AKI Network (AKIN) classification to the Kidney Disease Improving Global Outcomes (KDIGO) classification [9-11]. A multinational epidemiological study using KDIGO criteria showed that the incidence of AKI in the ICU was 57.3\% [12], which implies concern regarding AKI the in ICU globally with extremely high morbidity reported. It is essential for physicians, researchers, and health policy makers to establish an accurate incidence of AKI [12]. There have been were large epidemiological studies of patients hospitalized with AKI in the Chinese population [13, 14]. However, there have been limited epidemiological data on AKI in the ICU in mainland China reported [15]. Therefore we performed a cohort study of all the adult patients in 30 ICUs of 28 hospitals in

(C) The Author(s). 2019 Open Access This article is distributed under the terms of the Creative Commons Attribution 4.0 International License (http://creativecommons.org/licenses/by/4.0/), which permits unrestricted use, distribution, and 
Beijing to determine the incidence, risk factors, renal replacement therapy (RRT) practice, and the outcome of patients with AKI.

\section{Methods}

Study design

This is a multi-center prospective cohort study on the epidemiology of AKI in ICU patients in Beijing, China. A full list of the participating hospitals is provided in Additional file 1 . The study was registered with the Chinese Clinical Trial Registry (ChiCTR-ONC-11001875). Thirty ICUs in 28 teaching hospitals (two of the hospitals include two ICUs, respectively) in Beijing participated in this prospective observational study between March 1, 2012, and August 31, 2012 (a 6-month period). The Ethics Committee of Capital Medical University, Fuxing Hospital and all other participating hospitals approved the informed consent waiver due to the anonymous and non-interventional nature of the study (2010FXHEC-KY026, Additional file 2). Patients admitted to any participating ICU during the study period were included. We excluded (1) patients under 18 years old, (2) undergoing any kind of RRT within 3 months, (3) kidney transplantation within 3 months, (4) anticipated length of stay in the ICU (ICU-LOS) for less than $24 \mathrm{~h}$, and (5) readmission to the ICU during the study period.

\section{Definitions}

AKI was defined by the KDIGO criteria [16]. Patients were categorized on the basis of serum creatinine and/or urine output; the criteria leading to the worst classification used. Baseline serum creatinine was defined as the lowest serum level during the preceding 3 months [17]. For patients without a baseline serum creatinine laboratory test result, the baseline was estimated by the modification of diet in renal disease (MDRD) equation and customized for the Chinese population, assuming a glomerular filtration rate (GFR) of $75 \mathrm{~mL} / \mathrm{min}$ per $1.73 \mathrm{~m}^{2}$ [18]. Sepsis was defined as the combination of infection and systemic inflammatory response syndrome [19].

\section{Data collection}

Data were recorded on the case-reported form (CRF) (Additional file 3). On admission, data regarding demographics, admission time point, in-hospital location before ICU admission, acute physiology age and chronic health evaluation II (APACHE II) score, simplified acute physiology score II (SAPS II) score, sequential organ failure assessment (SOFA) score, baseline serum creatinine, comorbidity, and medications administrated before ICU admission were collected. During the first 10 days after admission, daily vital signs, urine output per hour, daily fluid balance, serum creatinine, medications, interventions, possible causes for AKI, diagnosis and stage of AKI, detailed information of RRT including reasons for initiation of RRT, mode of RRT, and anticoagulant and dilution patterns were collected. Diagnosis of sepsis and association between sepsis and AKI were reported. Outcome measures were collected including mortality, ICU-LOS, costs, withholding or withdrawal of life-sustaining treatments (WH/WD), and RRT dependence on the 7th and 28th days.

\section{Statistical analyses}

Continuous variables are presented as medians with interquartile ranges (IQRs), and compared by the MannWhitney U-test or Kruskal-Wallis ANOVA test. Categorical variables were compared using either the chi-square test or Fisher's exact test when appropriate. Statistical descriptions and tests above were performed using SPSS version 17.0.1 (SPSS Inc., Chicago, IL, USA). Multivariable logistic regression with odds ratio (OR) and 95\% confidence interval $(\mathrm{CI})$ was performed to assess independent risk factors for AKI development. Cox proportional hazards regression analysis with hazard ratio (HR) and 95\% CI was performed to examine whether the KDIGO stage was associated with mortality adjustment for baseline severity of illness, age and other factors. We used weighted estimators corresponding to each covariate derived from the fited logistic and Cox regression coefficients. The prognostic index was calculated by summing the number of risk points corresponding to each weighted covariate used to build the two nomograms. The specific codes used are provided in Additional file 4: Appendix 4. A $p$ value of less than 0.05 was considered significant. The nomograms of logistic regression and Cox regression were performed using R 3.5.1. The function "lrm" of the package "rms" was used for the logistic regression. The function "cph" of the package "survival" was used for the Cox regression.

\section{Results}

A total of 3107 participants were included in the final analysis among the 9049 patients admitted to the participating ICUs during the study period. The flow chart is presented in Fig. 1. In comparison of the characteristics between patients with and without AKI, patients with AKI have a higher median age, baseline serum creatinine, APACHE II score, SAPS II score, SOFA score, nonrenal SOFA score, more comorbidity, a higher rate of mechanical ventilation, use of inotropic agents and diuretics, and WH/WD. (Table 1).

\section{Incidence of AKI}

The incidence of AKI was 51.0\% (1584 of 3107) including stage 1 AKI 23.1\% (718 of 3107), stage 2 AKI 11.8\% (368 of 3107), and stage 3 AKI 15.7\% (498 of 3107) (Fig. $1)$. The majority of patients with AKI $(87.6 \%, 1388$ of 1584) developed AKI during the first 4 days after admission to the ICU (Fig. 2). 


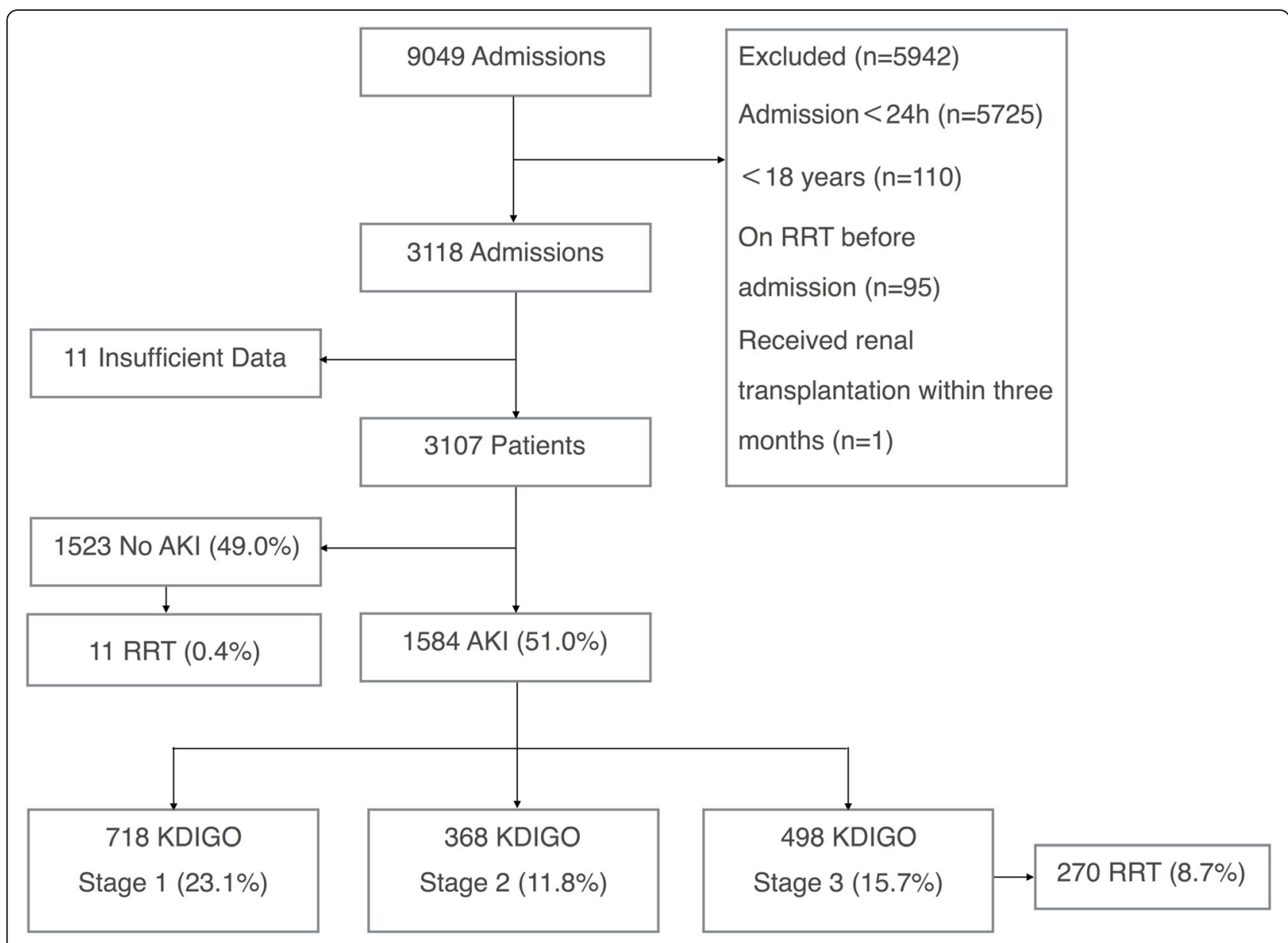

Fig. 1 Flow chart. AKI acute kidney injury, KDIGO Kidney Disease: Improving Global Outcomes, RRT renal replacement therapy

\section{Causes and risk factors of AKI}

Hypovolemia (25.4\%), sepsis on ICU admission (22.2\%) and low cardiac output (20.5\%) were the top three possible causes. The logistic regression nomogram indicated that baseline creatinine $(\mathrm{OR}=1.00$; 95\% CI 1.00-1.01), APACHE II score (OR $=1.05$; $95 \%$ CI: 1.04-1.07), SOFA score $(\mathrm{OR}=1.16 ; 95 \%$ CI 1.13-1.19), sepsis $(\mathrm{OR}=1.88 ; 95 \% \mathrm{CI}$ 1.56-2.27) and exposure to nephrotoxic drugs $(\mathrm{OR}=1.41$; 95\% CI 1.19-1.66) might be independent predictors of AKI development (Fig. 3a). There were 876 patients diagnosed with sepsis on ICU admission and/or during ICU stays. Physicians reported that 296 (33.8\%) cases of sepsis contributed to AKI, and 175 (20.0\%) cases of sepsis were possibly associated with AKI development.

\section{Renal replacement therapy}

A total of 281 patients were treated with RRT, including 270 patients with AKI (accounting for $17 \%$ of the AKI and $8.7 \%$ of all patients) and 11 non-AKI patients. The top four reasons to initiate RRT were anuria/oliguria $(71.9 \%, 201 /$ $281)$, severe metabolic acidosis $(25.6 \%, 72 / 281)$, hyperkalemia $(21.7 \%, 61 / 281)$ and fluid overload $(20.3 \%, 57 / 281)$.
Furthermore, 38.3\% (109 of 281) of patients had multiple reasons for initiating RRT, with anuria/oliguria together with hyperkalemia being most commonly reported. The 11 non-AKI patients received RRT due to acute heart failure ( $n=4$, to reduce heart load), poisoning ( $n=3$, to detoxify), heat stroke ( $n=2$, for rapid hypothermia), and sever e sepsis $(n=2$, to clear inflammatory media). The characteristics and outcomes of RRT patients are presented in Table 2 . The majority of RRT procedures were continuous RRT (CRRT). Intermittent RRT (IRRT) was seldom used (2\%). Continuous veno-venous hemofiltration $(\mathrm{CVVH})$ was the most common mode. Among 277 patients with the modes of anticoagulant reported, sodium citrate $(121,43.7 \%)$ was the most commonly reported anticoagulation pattern, followed by no anticoagulation $(68,24.5 \%)$, unfractionated heparin $(53,19.1 \%)$ and lowmolecular-weight heparin (22, 7.9\%). For dilution patterns reported in 269 cases, predilution, combination of preand post-dilution, and postdilution accounted for 56.5, 25.3 , and $18.2 \%$, respectively. Of the 284 reported catheter insertion sites, the femoral vein was the most common (74.3\%, 211/284; with 125 right femoral vein, 86 left 
Table 1 Characteristics and outcomes of included patients

\begin{tabular}{|c|c|c|c|c|}
\hline & All patients & AKI & Non-AKI & $p$ \\
\hline Number of patients & 3107 (100\%) & $1584(50.9 \%)$ & $1523(49.1 \%)$ & \\
\hline Age (years) & $64(51-77)$ & $67(53-78)$ & $62(49-74)$ & $<0.001$ \\
\hline Male & $1912(61.5 \%)$ & $970(61.2 \%)$ & $942(61.9 \%)$ & 0.74 \\
\hline Baseline SCr & $77(60.2-93)$ & 79 (63-94) & $74(58.8-89)$ & $<0.001$ \\
\hline APACHE I| score & $14(10-20)$ & $17(12-23)$ & $12(8-16)$ & $<0.001$ \\
\hline SAPS II score & $34(26-45)$ & $39(30-52)$ & $29(23-38)$ & $<0.001$ \\
\hline SOFA score & $6(3-8)$ & $7(4-10)$ & $4(2-7)$ & $<0.001$ \\
\hline Non-renal SOFA score & $5(3-8)$ & $6(4-9)$ & $4(2-6)$ & $<0.001$ \\
\hline \multicolumn{5}{|l|}{ Co-morbidity } \\
\hline $\mathrm{CHD}$ & 615 (19.8\%) & $370(23.4 \%)$ & $245(16.1 \%)$ & $<0.001$ \\
\hline CHF (NYHA IV) & $217(7.0 \%)$ & $152(9.6 \%)$ & $65(4.3 \%)$ & $<0.001$ \\
\hline $\mathrm{HT}$ & $1222(39.3 \%)$ & $687(43.4 \%)$ & 535 (35.1\%) & $<0.001$ \\
\hline DM & $532(17.1 \%)$ & $320(20.2 \%)$ & $212(13.9 \%)$ & $<0.001$ \\
\hline COPD & $166(5.3 \%)$ & $98(6.2 \%)$ & $68(4.5 \%)$ & 0.038 \\
\hline CKD & $203(6.5 \%)$ & $167(10.5 \%)$ & $36(2.4 \%)$ & $<0.001$ \\
\hline CLF & $91(2.9 \%)$ & $53(3.3 \%)$ & $38(2.5 \%)$ & 0.168 \\
\hline \multicolumn{5}{|l|}{ Sources of patients } \\
\hline ED & 774 (24.9\%) & $440(27.8 \%)$ & $334(21.9 \%)$ & $<0.001$ \\
\hline general wards & $586(18.9 \%)$ & $383(24.2 \%)$ & $203(13.3 \%)$ & $<0.001$ \\
\hline post-operation & $1627(52.4 \%)$ & $692(43.7 \%)$ & 935 (61.4\%) & $<0.001$ \\
\hline other ICUs & $31(1.0 \%)$ & $21(1.3 \%)$ & $10(0.7 \%)$ & $<0.001$ \\
\hline other hospitals & $89(2.9 \%)$ & $48(3.0 \%)$ & $41(2.7 \%)$ & $<0.001$ \\
\hline \multicolumn{5}{|l|}{ Medications before admission } \\
\hline Aminoglycosides & $47(1.5 \%)$ & $30(1.9 \%)$ & $17(1.1 \%)$ & 0.079 \\
\hline Glycopeptide antibiotics & $50(1.6 \%)$ & $32(2.0 \%)$ & $18(1.2 \%)$ & 0.065 \\
\hline Radio-contrast media & $560(18 \%)$ & $305(19.3 \%)$ & $255(16.7 \%)$ & 0.069 \\
\hline Mannitol & $92(3.0 \%)$ & $45(2.8 \%)$ & 47 (3.1\%) & 0.751 \\
\hline NSAIDs & $253(8.1 \%)$ & $152(9.6 \%)$ & $101(6.6 \%)$ & 0.003 \\
\hline ACEI/ARB & $523(16.8 \%)$ & $281(17.1 \%)$ & $242(15.9 \%)$ & 0.179 \\
\hline Statin & $352(11.3 \%)$ & $184(11.6 \%)$ & $168(11.0 \%)$ & 0.611 \\
\hline $\mathrm{SCr}$ & $83(62-116)$ & $105.4(72-161)$ & $70.8(56.7-89)$ & $<0.001$ \\
\hline Sepsis & $641(20.6 \%)$ & $459(29.0 \%)$ & $182(12.0 \%)$ & $<0.001$ \\
\hline \multicolumn{5}{|l|}{ Organ failure } \\
\hline Respiratory failure & $811(26.1 \%)$ & $538(34.0 \%)$ & $273(17.9 \%)$ & $<0.001$ \\
\hline Acute hepatic failure & $53(1.7 \%)$ & $37(2.3 \%)$ & $16(1.1 \%)$ & 0.006 \\
\hline Hematologic failure & $44(1.4 \%)$ & $34(2.1 \%)$ & $10(0.7 \%)$ & $<0.001$ \\
\hline Shock & $484(15.6 \%)$ & $353(22.3 \%)$ & $131(8.6 \%)$ & $<0.001$ \\
\hline Cardiogenic shock & 117 (3.8\%) & $96(6.1 \%)$ & $21(1.4 \%)$ & $<0.001$ \\
\hline Hypovolemic shock & $201(6.5 \%)$ & $130(8.2 \%)$ & $71(4.7 \%)$ & 0.004 \\
\hline Septic shock & $222(7.1 \%)$ & $173(10.9 \%)$ & 49 (3.2\%) & $<0.001$ \\
\hline Obstructive shock & $4(0.1 \%)$ & $2(0.1 \%)$ & $2(0.1 \%)$ & $<0.001$ \\
\hline MV & $2344(75.4 \%)$ & $1260(79.5 \%)$ & $1084(71.2 \%)$ & $<0.001$ \\
\hline Vasopressors & $930(29.9 \%)$ & $480(30.3 \%)$ & $450(29.5 \%)$ & 0.666 \\
\hline Inotropic agents & $665(21.4 \%)$ & $366(23.1 \%)$ & 299 (19.6\%) & 0.02 \\
\hline
\end{tabular}


Table 1 Characteristics and outcomes of included patients (Continued)

\begin{tabular}{lllll}
\hline & All patients & AKI & Non-AKI & $p$ \\
\hline Diuretics & $1650(53.1 \%)$ & $1067(67.4 \%)$ & $583(38.3 \%)$ & $293(19.2 \%)$ \\
WH/WD & $691(22.2 \%)$ & $398(25.1 \%)$ & $49(3.2 \%)$ & $<0.001$ \\
ICU mortality & $395(12.7 \%)$ & $346(21.8 \%)$ & $104(6.8 \%)$ & $<.001$ \\
28-day mortality & $542(17.4 \%)$ & $438(27.7 \%)$ & $3(2-6)$ & $<0.001$ \\
ICU-LOS (days) & $4(2-10)$ & $5.5(3-11)$ & $26,000(14000-51,000)$ & $<0.001$ \\
ICU overall costs (RMB) & $32,000(17000-74,000)$ & $42,000(21000-95,000)$ & 6.001 \\
ICU daily costs (RMB) & $6500(4500-10,000)$ & $6667(4826-10,182)$ & 6.001 & $<0.001$ \\
\hline
\end{tabular}

Values are presented as $\mathrm{n}$ (proportion) or median (interquartile range)

AKI Acute kidney injury, SCr Serum creatinine, CHD Coronary heart disease, CHF Chronic heart failure, NYHA the New York heart association functional

classification, HT Hypertension, DM Diabetes mellitus, COPD Chronic obstructive pulmonary disease, CKD Chronic kidney disease, CLF Chronic liver failure, APACHE II Acute physiology and chronic health evaluation II, SAPS II Simplified acute physiology score II, SOFA Sequential organ failure assessment score non-renal, SOFA Sequential organ failure assessment score without the renal component, ED Emergency department, ICU Intensive care unit, NSAIDs Non-steroidal antiinflammatory drugs, $A C E I$ Angiotensin-converting enzyme inhibitor, $A R B$ Angiotensin receptor blocker, $M V$ Mechanical ventilation, WH/WD Withholding or withdrawal of life-sustaining therapy

femoral vein), followed by the internal jugular vein $(23.6 \%$, 67/284; with 48 right jugular vein, 19 left jugular vein), 5 subclavian vein and 1 arterio-venous fistula. Continuous RRT with predilution, citrate for anticoagulation and femoral vein for vascular access was the most common pattern (29.9\%, 84 of 281). For adverse events, bleeding or oozing at the catheterization site was the most commonly reported $(12.5 \%, 35$ of 281$)$, followed by gastrointestinal bleeding (9.6\%, 27 of 281) and cutaneous bruise or ecchymoses $(6.8 \%, 19$ of 281$)$. The 28 -day mortality of patients treated with RRT was $48.8 \%$. In 281 patients treated with RRT, $76.0 \%$ (173 of 220 survivors) of patients depended on RRT on the 7th day. Among 144 survivors on the 28th day, 41 patients (28.5\%) were dependent on RRT; 25 (19.7\%) patients depended on intermittent hemodialysis (IHD), and 16 (11.1\%) depended on CRRT.

\section{No. of AKI onset $\quad$ Total AKI patients}

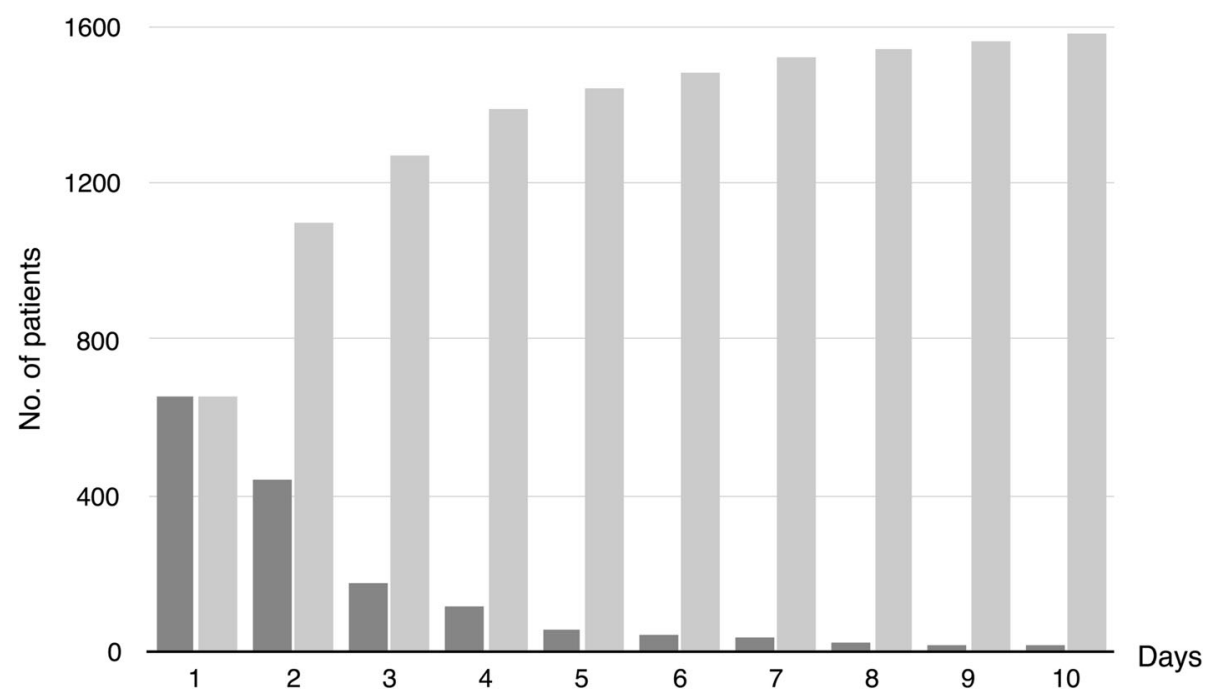

\begin{tabular}{|c|c|c|c|c|c|c|c|c|c|c|}
\hline Day & 1 & 2 & 3 & 4 & 5 & 6 & 7 & 8 & 9 & 10 \\
\hline No. of AKI onset & 651 & 444 & 177 & 116 & 54 & 43 & 34 & 26 & 19 & 20 \\
\hline Total AKI patients & 651 & 1095 & 1272 & 1388 & 1442 & 1485 & 1519 & 1545 & 1564 & 1584 \\
\hline
\end{tabular}

Fig. 2 Daily AKI onset and accumulative AKI patients on the first 10 days. No. number, AKI acute kidney injury, CRF case report form, KDIGO the Kidney Disease: Improving Global Outcomes 

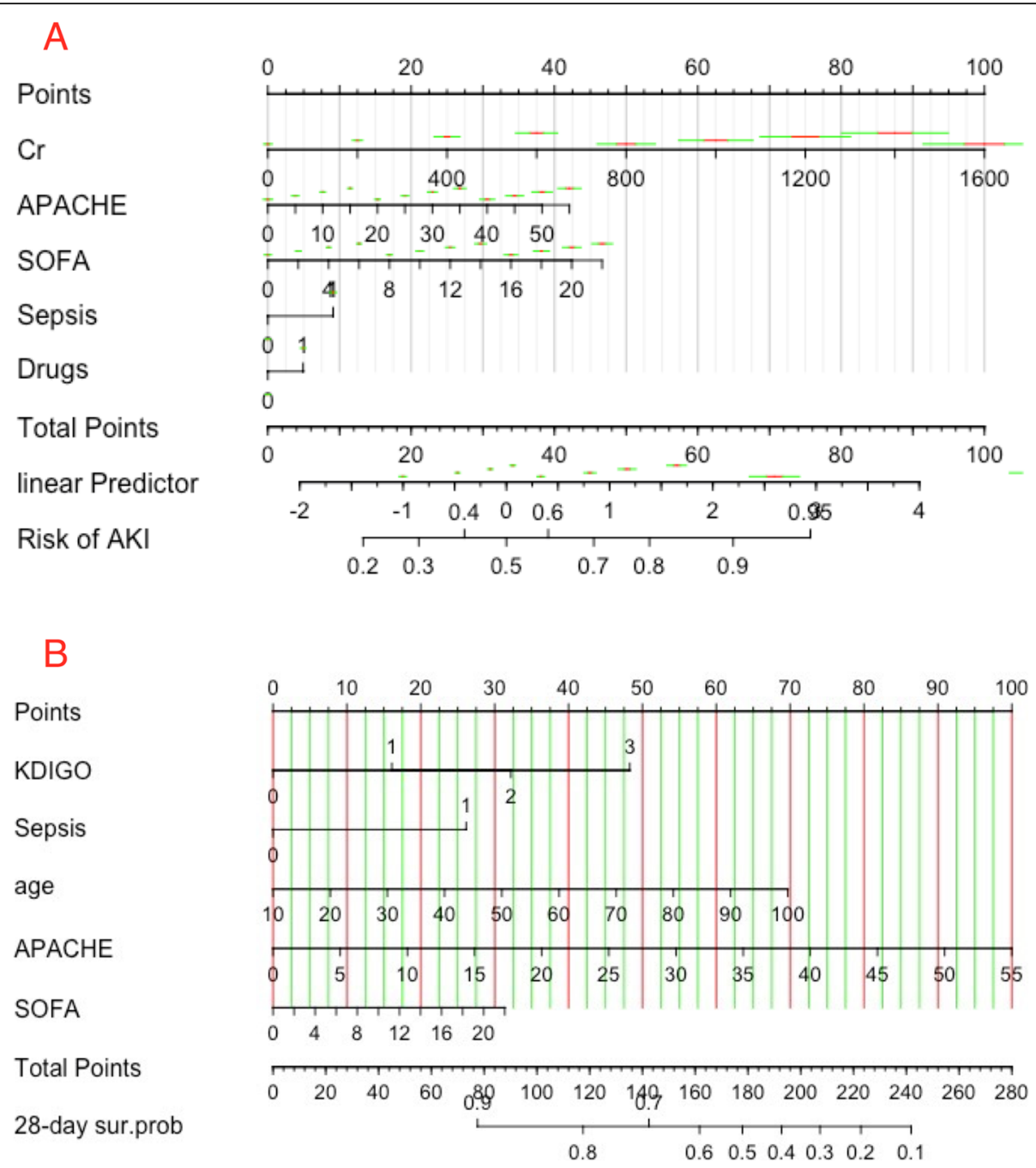

Fig. 3 Nomogram of independent predictors for development of AKI and 28 day mortality. Each predictor with a given value can be mapped to the Points axis. The sum of these points can be referred to in the Total Points axis. Then the linear predictor and the probability of development of AKI (A) and survival (B) can be obtained from corresponding axis. The green bar indicates the 0.7 confidence limits for each score, and the short red bar corresponds to 0.1 confidence limits. Cr baseline creatinine, APACHE acute physiology and chronic health evaluation II, SOFA sequential organ failure assessment score, Drugs nephrotoxic drug exposure, KDIGO Kidney Disease: Improving Global Outcomes criteria and stage of AKI, 28-day sur.pro probability of survival on the 28th day

The ICU-LOS, mortality rate and costs of RRT patients were significantly higher than those of non-RRT AKI patients (Table 2).

\section{Clinical outcomes and costs}

Patients with AKI had a significantly higher rate of withholding or withdrawing of life-sustaining treatments, ICU mortality, 28-day mortality, longer ICU-LOS, and higher ICU overall costs (Table 1). The 28-day mortality rate of non-AKI patients was $6.83 \%$. The mortality rates of AKI stages $1-3$ were $15.04,27.99$, and $45.18 \%$, respectively. The 28-day mortality rate of the 917 septic AKI patients was $36.5 \%$. The Cox regression nomogram indicated that a higher stage of AKI ( $\mathrm{HR}=1.35$; 95\% CI 1.25-1.47), higher age ( $\mathrm{HR}=1.01 ; 95 \% \mathrm{CI} 1.01-1.02)$, high APACHE II (HR = 1.03; 95\% CI 1.02-1.05) and SOFA scores (HR $=1.03$; $95 \%$ CI 1.00-1.06), and sepsis ( $\mathrm{HR}=1.63 ; 95 \%$ CI1.35-1.98) were independent predictors of mortality (Fig. 3b).

\section{Discussion}

Our results indicated a high incidence of AKI in the ICU. Approximately half of our ICU patients experienced AKI. The incidence in the present study was significantly higher than those in an international epidemiological study in 2005 [20] and the FINNAKI study [21], which might be attributed to the discrepancy in diagnostic criteria. A study comparing the three diagnostic criteria indicated that the KDIGO criteria identified more patients with AKI 
Table 2 Characteristics and outcomes of RRT patients

\begin{tabular}{llll}
\hline & RRT & Non-RRT & \\
\hline $\begin{array}{l}\text { Number of patients } \\
\text { Characteristics }\end{array}$ & 281 & 1314 & \\
Age & $66(52-79)$ & $67(53-78)$ & 0.141 \\
Male & $178(63.3 \%)$ & $791(60.2 \%)$ & $<0.05$ \\
APACHE II & $22(17-29)$ & $16(11-22)$ & $236(17.9 \%)$ \\
ICU mortality & $109(38.8 \%)$ & $303(23.0 \%)$ & $<0.05$ \\
$28-$ day mortality & $130(46.3 \%)$ & $5(3-10)$ & $<0.05$ \\
ICU-LOS (days) & $8(5-17)$ & $37,000(19000-87,000)$ & $<0.05$ \\
ICU overall costs (RMB) & $104,000(60000-213,000)$ & $6143(4333-9000)$ & $<0.05$ \\
ICU daily costs (RMB) & $9765(5580-14,625)$ & NR & $<0.05$ \\
RRT dependent on 7th day & $173 / 220(78.6 \%)$ & NR & $<0.05$ \\
RRT dependent on 28th day & $41 / 144(28.5 \%)$ & $<0.05$ \\
\hline APACHE II Acute physiology and chronic health evaluation 11 ICU Intensive care unit, LOS Length of stay, NR Not recorded, RRT Renal replacement therapy
\end{tabular}

and were more predictive of short-term mortality [22]. The incidence in the present study was similar to the Acute Kidney Injury- Epidemiological Prospective Investigation (AKI-EPI) study [12]. The AKI-EPI study [12] was the first multinational epidemiological study in the ICU using the KDIGO criteria. Both AKI-EPI and our study demonstrated an unsatisfactory prevention and increasing burden of AKI. In our study, the top three possible causes reported by physicians were hypovolemia, sepsis on ICU admission and low cardiac output, which was similar to the result of the FINNAKI study (preceding AKI severe sepsis, pre-ICU hypovolemia and pre-ICU hypotension) [21]. Our logistic regression showed comprehensible risk factors. The RRT ratio in our study was similar to that of the FINNAKI study (8.6\% vs 9.4\%) [21], but lower than that of the AKI-EPI study (13.5\%) [12]. The higher RRT ratio in the AKI-EPI study could be attributable to the higher ratio (KDIGO stage 3: 30.0\%) of patients with more severe AKI in comparison with the other two studies (KDIGO stage 3: 15.7\%; AKIN stage 3: 14.1\%). The RRT pattern results indicated that CRRT was mainly chosen (97.9\%) and that IRRT was seldom used. A previous observational study in French ICUs showed that 40\% of RRT patients had CRRT and 60\% had IRRT [23], which varied greatly from our results. Although the previous studies have shown no difference in clinical outcomes comparing CRRT and IRRT in the ICU [24-26], our results revealed a strong inclination to CRRT in our ICUs. The advantages of CRRT include better hemodynamic tolerance, accurate balance control and better clearance of the middle molecule $[11,27]$. Besides our ICU physicians and nurses were familiar with CRRT and unfamiliar with IRRT. Our results showed that the femoral vein $(74.6 \%)$ was the most common catheter location, differing from the recommendations in the guideline (the right internal jugular vein) [28]. Studies have suggested that the internal jugular vein might be preferable to the femoral vein to minimize dialysis catheter dysfunction and blood recirculation to improve RRT provision and reduce the risk of infection [29-32]. The reasons for our physicians preferring femoral access might be the advantages of convenience, efficiency and fewer complications in the operating procedure. The results indicated that citrate was the most commonly used for anticoagulant. Meta-analyses [33-35] suggested that citrate is preferable to heparin in anticoagulation for CRRT to prolong circuit life span, reduce the risk of bleeding [34, 35], and increase the delivered RRT dose [33]. Citrate is novel and has not been used for long in CRRT; nevertheless, our results indicated that it has already been widely used in Beijing. However, considering the advantages of better medical resources in Beijing, the capital city of China, the result may not be representative of the whole country. The distinction of guideline compliance with respect to vascular access and anticoagulation might imply that changes in medication are more acceptable for physicians than are changes in techniques. The mortality rate was comparable to the FINNAKI study [21]. The regression analysis indicated that a greater severity of $\mathrm{AKI}$, and comorbidity of sepsis were associated with an increased risk of mortality, which was in accordance with the previous studies $[12,13,21]$. Our results showed a significantly higher ICU costs for RRT patients, which is reasonable and comparable to other studies [26, 35]. RRT patients had a higher AKI stage, more complications, higher disease severity score and longer ICU-LOS, all of which lead to higher costs [35]. Our results showed that increased AKI stage and septic AKI were associated with higher mortality, and those trends were also found in a study that evaluated patients with AKI with and without sepsis [36]. More than $1 / 4$ of survivors were RRT dependent, which would lead to heavy burdens. There are strengths in our study. This is the first large, prospective, 
multicenter cohort study of AKI in the ICU in Beijing. We used the KDIGO criteria to evaluate the prevalence of AKI. Previous studies [22, 37, 38] indicated that the KDIGO criteria defined more patients with AKI in comparison with RIFLE and AKIN. Further analysis showed that the patients missed by RIFLE had higher mortality rate and longer hospital-LOS than the patients missed by KDIGO [22]. For the first time the incidence of AKI in the ICU for ten consecutive days was reported, to the best of our knowledge. The results revealed an obvious downtrend of AKI onset over time. We consider this result to be meaningful epidemiological data that might imply the necessity of key vigilance against the risk of AKI in the first 4 days after ICU admission. Furthermore, we investigated the comprehensive situation in RRT practice. Thus, we understood our insufficiencies and underlying causes, which are important areas for policy makers and physicians to make improvements. There were limitations in our study. First, our participating hospitals were all located in Beijing. As the capital city of China, Beijing enjoys better medical resources. Thus, the results of our study might not be representative of hospitals nationwide. Second, we used the MDRD equation to estimate the baseline serum creatinine for missing values, as recommended [18, 39]. However, the MDRD method may result in under- or overestimation of baseline creatinine [39, 40]. Third, we screened patients for AKI for the first 10 days after admission to the ICU. Thus, we were unable to analyze lateronset AKI. However, based on our results, the majority of patients with AKI (87.6\%) had AKI onset in the first 4 days after admission. Our data on the occurrence of AKI during the first 10 days may imply that the onset of AKI in the ICU decreases over time, and new onset after 10 days would be minimal.

\section{Conclusions}

There was a high incidence of AKI in the ICU. Approximately half of our ICU patients experienced AKI. The majority of patients with AKI developed AKI during the first four ICU days. For RRT patterns, continuous RRT, predilution, citrate, and femoral vein were the most commonly used RRT procedure, dilution mode, anticoagulant and vascular access, respectively. AKI was associated with increased mortality and costs, incomplete kidney recovery and a series of adverse outcomes. Higher AKI stage, septic-AKI and the need for RRT were associated with increased mortality.

\section{Supplementary information}

Supplementary information accompanies this paper at https://doi.org/10. 1186/s12882-019-1660-z

Additional file 1. Full list of participating hospitals.
Additional file 2. Ethical approval documents and all other ethical bodies that approved our study in the various centers involved.

Additional file 3. CRF in Chinese and outline in English.

Additional file 4. Code for nomograms.

\section{Abbreviations}

AKI: Acute Kidney Injury; AKIN: Acute Kidney Injury Network; APACHE II: Acute Physiology Age and Chronic Health Evaluation II; Cl: Confidence interval; CKD: Chronic Kidney Disease; CRF: Case Reported Form; CRRT: Continuous Renal Replacement Therapy; CWH: Continuous VenoVenous Hemofiltration; GFR: Glomerular Filtration Rate; HR: Hazard Ratio; ICU: Intensive Care Unit; IHD: Intermittent Hemodialysis; IQR: Interquartile Range; IRRT: Intermittent Renal Replacement Therapy; KDIGO: Kidney Disease Improving Global Outcomes; LOS: Length Of Stay; MDRD: Modification of Diet in Renal Disease; OR: Odds Ratio; RIFLE: Risk, Injury, Failure, Loss, Endstage; RRT: Renal Replacement Therapy; SAPS II: Simplified Acute Physiology Score II; SOFA: Sequential Organ Failure Assessment; WH/WD: Withholding or Withdrawal of Life-sustaining Treatments

\section{Acknowledgements}

Members of the BAKIT group: Yuan Xu (Department of Critical Care Medicine, Beijing Tongren Hospital, Capital Medical University); Jianxin Zhou (jianxinz@yeah.net), Department of Critical Care Medicine, Beijing Tiantan Hospital affiliated to Capital Medical University); Ang Li (Department of Critical Care Medicine, Beijing Friendship Hospital, Capital Medical University); Jingyuan Liu (Department of Critical Care Medicine, Beijing Ditan Hospital , Capital Medical University); Wenxiong Li (Surgical Intensive Care Unit, Beijing Chaoyang Hospital , Capital Medical University); Wenjin Chen (Neurological intensive care unit, Xuanwu Hospital, Capital Medical University); Jianguo Jia (Surgical Intensive Care Unit, Xuanwu Hospital, Capital Medical University); Xi Zhu (Department of Critical Care Medicine, Peking University Third Hospital); Penglin Ma (Department of Critical Care Medicine, The 309th Hospital of Chinese People's Liberation Army); Wei Chen (Department of Critical Care Medicine, Beijing Shijitan Hospital, Capital Medical University); Dongxin Wang (Department of Critical Care Medicine, Peking University First Hospital); Youzhong An (Department of Critical Care Medicine, Peking University People's Hospital); Qingyuan Zhan (Department of Critical Care Medicine, China-Japan Friendship Hospital); Gang Li (Department of Critical Care Medicine, China-Japan Friendship Hospital); Haitao Zhang (Surgical Intensive Care Unit, Fuwai Hospital, China Academy of Medical Science and Peking Union Medical College); Bo Ning (Department of Critical Care Medicine, Air Force General Hospital of Chinese People's Liberation Army); Zhongjie He (Department of Critical Care Medicine, The First Affiliated Hospital of General Hospital of People's Liberation Army); Zhicheng Zhang (Department of Critical Care Medicine, Navy General Hospital); Yaxiong Sun (Department of Critical Care Medicine, The Luhe Teaching Hospital of the Capital Medical University); Shijie Jia (Surgical Intensive Care Unit, Beijing Anzhen Hospital, Capital Medical University); Yalin Liu (Surgical Intensive Care Unit, Beijing Hospital); Rui Cheng (Department of Critical Care Medicine, General Hospital of Armed Police Forces); Qing Song (Department of Critical Care Medicine, The General Hospital of People's Liberation Army); Jinning Liu (Surgical Intensive Care Unit, Beijing YouAn Hospital, Capital Medical University); Yangong Chao (Department of Critical Care Medicine, Hua Xin Hospital, First Hospital of Tsinghua University); Huizhen Li (Department of Critical Care Medicine, Beijing Shunyi Hospital of China Medical University); Li Feng (Department of Critical Care Medicine, Beijing Geriatric Hospital); Ruochun Shi (Department of Critical Care Medicine, Beijing No.6 Hospital); Xiuming Xi, Li Jiang, Ying Wen, Bo Zhu, Meiping Wang, Qi Jiang,Peng Wang, Yujie Deng, Yan Sun, Yanyan Yin, Xin Zhang, Li Zhang, Zhen Zhao, Ying Wang, Ran Lou, Jing Wang (Department of Critical Care Medicine, Fuxing Hospital, Capital Medical University).

\section{Authors' contributions}

$J$ and $X X M$ designed the study. ZYB and LXY drafted the manuscript. WY, WMP, ZZ, YYY, ZB have made substantial contributions to the acquisition of the data. DB substantively revised the manuscript. All authors read and approved the manuscript.

\section{Funding}

This study was supported by a grant from the Beijing Municipal Science \& Technology Commission, a government fund used to improve health care 
quality (number D101100050010058). It offered financial support for data collection. The funders had no role in study design, data analysis, interpretation of data or preparation of the manuscript.

\section{Availability of data and materials}

The dataset used and analyzed during the current study is available from the corresponding author on reasonable request.

\section{Ethics approval and consent to participate}

The Ethics Committee of Capital Medical University, Fuxing Hospital and all other participating hospitals approved the informed consent waiver due to the anonymous and non-interventional nature of the study (Additional file 2).

\section{Consent for publication}

Not applicable.

\section{Competing interests}

The authors declare that they have no competing interests.

\section{Author details}

'Department of Critical Care Medicine, Fuxing Hospital, Capital Medical University, 20A Fuxingmenwai Street, Xicheng District, Beijing 100038, China. ${ }^{2}$ Medical Research and Biometrics Center, Fuwai Hospital, National Center for Cardiovascular Diseases, Peking Union Medical College, Chinese Academy of Medical Sciences, Room 101-106, Block A, Shilong West Road, Mentougou District, Beijing 102300, China. ${ }^{3}$ Department of Critical Care Medicine, Tiantan Hospital, Capital Medical University, 119 Nansihuanxi Road, Fengtai District, Beijing 100070, China. ${ }^{4}$ Departmrnt of General Practice, Beitaipingzhuang Community Health Service Center, Building 6, Wenhuiyuan, Wenhuiyuan South Road, Haidian District, Beijing 100082, China. ${ }^{5}$ Medical ICU, Peking Union Medical College Hospital, Peking Union Medical College and Chinese Academy of Medical Sciences, 1 Shuai Fu Yuan, Beijing 100730, China. ${ }^{6}$ Department of Epidemiology and Health Statistics, School of Public Health, Capital Medical University, 10 Xitoutiao, Youanmenwai, Fengtai District, Beijing 100069, China. ${ }^{7}$ Center of urology and metabolism, Beijing Rehabilitation Hospital, Capital Medical University, Xixiazhuang, Badachu Road, Shijingshan District, Beijing 100114, China.

Received: 22 March 2019 Accepted: 5 December 2019

Published online: 16 December 2019

\section{References}

1. Lameire NH, Bagga A, Cruz D, De Maeseneer J, Endre Z, Kellum JA, Liu KD, Mehta RL, Pannu N, Van Biesen W, et al. Acute kidney injury: an increasing global concern. Lancet. 2013;382:170-9.

2. Ostermann M, Chang RW. Acute kidney injury in the intensive care unit according to RIFLE. Crit Care Med. 2007;35:1837-43.

3. Singbartl K, Kellum JA. AKI in the ICU: definition, epidemiology, risk stratification, and outcomes. Kidney Int. 2012;81:819-25.

4. Bellomo R, Kellum JA, Ronco C. Acute kidney injury. Lancet. 2012;380:756-66.

5. Hoste EA, Schurgers M. Epidemiology of acute kidney injury: how big is the problem. Crit Care Med. 2015;36:S146-51.

6. Murugan R, Kellum JA. Acute kidney injury: what's the prognosis. Nat Rev Nephrol. 2011;7:209-17.

7. Chawla LS, Eggers PW, Star RA, Kimmel PL. Acute kidney injury and chronic kidney disease as interconnected syndromes. N Engl J Med. 2014;371:58-66.

8. Ostermann M, Joannidis M. Acute kidney injury 2016: diagnosis and diagnostic workup. Crit Care. 2016;20:299.

9. Bellomo R, Ronco C, Kellum JA, Mehta RL, Palevsky P. Acute Dialysis Quality Initiative workgroup. Acute renal failure: definition, outcome measures, animal models, fluid therapy and information technology needs: the second international consensus conference of the acute dialysis quality initiative (ADQI) group. Crit Care. 2004;8:R204-12.

10. Mehta RL, Kellum JA, Shah SV, Molitoris BA, Ronco C, Warnock DG, Levin A. Acute Kidney Injury Network Acute kidney injury network: report of an initiative to improve outcomes in acute kidney injury. Crit Care. 2007;11:R31.

11. Kidney Disease: Improving Global Outcomes (KDIGO) Acute Kidney Injury Work Group. KDIGO clinical practice guideline for acute kidney injury. Kidney Int. 2012;2:1-138.
12. Hoste EA, Bagshaw SM, Bellomo R, Cely CM, Colman R, Cruz DN, Edipidis K, Forni LG, Gomersall CD, Govil D, et al. Epidemiology of acute kidney injury in critically ill patients: the multinational AKI-EPI study. Intensive Care Med. 2015:41:1411-23.

13. Xu X, Nie S, Liu Z, Chen C, Xu G, Zha Y, Qian J, Liu B, Han S, Xu A, et al. Epidemiology and Clinical Correlates of AKI in Chinese Hospitalized Adults. Clin J Am Soc Nephrol. 2015;10:1510-8.

14. Yang L, Xing G, Wang L, Wu Y, Li S, Xu G, He Q, Chen J, Chen M, Liu X, et al. Acute kidney injury in China: a cross-sectional survey. Lancet. 2015;386: 1465-71.

15. Wen Y, Jiang L, Xu Y, Qian CY, Li SS, Qin TH, Chen EZ, Lin JD, Ai YH, Wu DW, et al. China Critical Care Clinical Trial Group (CCCCTG). Prevalence, risk factors, clinical course, and outcome of acute kidney injury in Chinese intensive care units: a prospective cohort study. Chin Med J. 2013;126:4409-16.

16. Kellum JA, Lameire N. KDIGO AKI Guideline Work Group. Diagnosis, evaluation, and management of acute kidney injury: a KDIGO summary (Part 1). Crit Care. 2013;17:204

17. Thongprayoon C, Cheungpasitporn W, Kittanamongkolchai W, Srivali N, Ungprasert P, Kashani K. Optimum methodology for estimating baseline serum creatinine for the acute kidney injury classification. Nephrology (Carlton). 2015:20:881-6.

18. Ma YC, Zuo L, Chen JH, Luo Q, Yu XQ, Li Y, Xu JS, Huang SM, Wang LN, Huang $W$, et al. Modified glomerular filtration rate estimating equation for Chinese patients with chronic kidney disease. J Am Soc Nephrol. 2006;17: 2937-44.

19. Bone RC, Balk RA, Cerra FB, Dellinger RP, Fein AM, Knaus WA, Schein RM, Sibbald WJ. Definitions for sepsis and organ failure and guidelines for the use of innovative therapies in sepsis. The ACCP/SCCM Consensus Conference Committee. Chest. 1992;101:1644-55.

20. Uchino S, Kellum JA, Bellomo R, Doig GS, Morimatsu H, Morgera S, Schetz M, Tan I, Bouman C, Macedo E, et al. Beginning and Ending Supportive Therapy for the Kidney (BEST Kidney) Investigators. JAMA. 2005;294:813-8.

21. Nisula S, Kaukonen KM, Vaara ST, Korhonen AM, Poukkanen M, Karlsson S, Haapio M, Inkinen O, Parviainen I, Suojaranta-Ylinen R, et al. Incidence, risk factors and 90-day mortality of patients with acute kidney injury in Finnish intensive care units: the FINNAKI study. Intensive Care Med. 2013:39:420-8.

22. Luo X, Jiang L, Du B, Wen Y, Wang M, Xi X. The Beijing Acute Kidney Injury Trial (BAKIT) workgroup: A comparison of different diagnostic criteria of acute kidney injury in critically ill patients. Crit Care. 2014;18:R144.

23. Rabindranath K, Adams J, Macleod AM, Muirhead N. Intermittent versus continuous renal replacement therapy for acute renal failure in adults. Cochrane Database Syst Rev. 2007;1:CD003773.

24. Bagshaw SM, Berthiaume LR, Delaney A, Bellomo R. Continuous versus intermittent renal replacement therapy for critically ill patients with acute kidney injury: a meta-analysis. Crit Care Med. 2008;36:610-7.

25. Srisawat N, Lawsin L, Uchino S, Bellomo R, Kellum JA. BEST Kidney Investigators: Cost of acute renal replacement therapy in the intensive care unit: results from The Beginning and Ending Supportive Therapy for the Kidney (BEST Kidney) study. Crit Care. 2010;14:R46.

26. Truche AS, Darmon M, Bailly S, Clec'h C, Dupuis C, Misset B, Azoulay E, Schwebel C, Bouadma L, Kallel $H$, et al. Continuous renal replacement therapy versus intermittent hemodialysis in intensive care patients: impact on mortality and renal recovery. Intensive Care Med. 2016:42:1408-17.

27. Lins RL, Elseviers MM, Van der Niepen P, Hoste E, Malbrain ML, Damas P, Devriendt J. SHARF investigators Intermittent versus continuous renal replacement therapy for acute kidney injury patients admitted to the intensive care unit: results of a randomized clinical trial. Nephrol Dial Transplant. 2009;24:512-8.

28. du Cheyron D, Bouchet B, Bruel C, Daubin C, Ramakers M, Charbonneau P. Antithrombin supplementation for anticoagulation during continuous hemofiltration in critically ill patients with septic shock: a case-control study. Crit Care. 2006;10:R45

29. Leblanc M, Fedak S, Mokris G, Paganini EP. Blood recirculation in temporary central catheters for acute hemodialysis. Clin Nephrol. 1996;45:315-9.

30. Liangos O, Rao M, Ruthazer R, Balakrishnan VS, Modi G, Pereira BJ, Jaber BL Factors associated with urea reduction ratio in acute renal failure. Artif Organs. 2004:28:1076-81.

31. Mrozek N, Lautrette A, Timsit JF, Souweine B. How to deal with dialysis catheters in the ICU setting. Ann Intensive Care. 2012:2:48.

32. Wu MY, Hsu YH, Bai CH, Lin YF, Wu CH, Tam KW. Regional citrate versus heparin anticoagulation for continuous renal replacement therapy: a metaanalysis of randomized controlled trials. Am J Kidney Dis. 2012;59:810-8. 
33. Liu C, Mao Z, Kang H, Hu J, Zhou F. Regional citrate versus heparin anticoagulation for continuous renal replacement therapy in critically ill patients: a meta-analysis with trial sequential analysis of randomized controlled trials. Crit Care. 2016;20:144

34. Bai M, Zhou M, He L, Ma F, Li Y, Yu Y, Wang P, Li L, Jing R, Zhao L, Sun S. Citrate versus heparin anticoagulation for continuous renal replacement therapy: an updated meta-analysis of RCTs. Intensive Care Med. 2015;41:2098-110.

35. Peters E, Antonelli M, Wittebole X, Nanchal R, François B, Sakr Y, Vincent IL, Pickkers P. A worldwide multicentre evaluation of the influence of deterioration or improvement of acute kidney injury on clinical outcome in critically ill patients with and without sepsis at ICU admission: results from The Intensive Care Over Nations audit. Crit Care. 2018;22:188.

36. Zeng X, McMahon GM, Brunelli SM, Bates DW, Waikar SS. Incidence, Outcomes, and Comparisons across Definitions of AKI in Hospitalized Individuals. Clin J Am Soc Nephrol. 2014:9:12-20.

37. Fujii T, Uchino S, Takinami M, Bellomo R. Validation of the Kidney Disease Improving Global Outcomes Criteria for AKI and Comparison of Three Criteria in Hospitalized Patients. Clin J Am Soc Nephrol. 2014;9:848-54.

38. Srisawat N, Kellum JA. Acute kidney injury: definition, epidemiology, and outcome. Curr Opin Crit Care. 2011;17:548-55.

39. Gaiao S, Cruz DN. Baseline creatinine to define acute kidney injury: is there any consensus. Nephrol Dial Transplant. 2010;25:3812-4.

40. Ricci Z, Cruz DN, Ronco C. Classification and staging of acute kidney injury: beyond the RIFLE and AKIN criteria. Nat Rev Nephrol. 2011;7:201-8.

\section{Publisher's Note}

Springer Nature remains neutral with regard to jurisdictional claims in published maps and institutional affiliations.

Ready to submit your research? Choose BMC and benefit from:

- fast, convenient online submission

- thorough peer review by experienced researchers in your field

- rapid publication on acceptance

- support for research data, including large and complex data types

- gold Open Access which fosters wider collaboration and increased citations

- maximum visibility for your research: over $100 \mathrm{M}$ website views per year

At $\mathrm{BMC}$, research is always in progress.

Learn more biomedcentral.com/submissions 